\title{
Key to successful hospital acquired pressure injury reduction: Leadership support and engagement
}

\author{
Sue Creehan, Joyce Black, Nick Santamaria, Jacqui Fletcher, Paulo Alves \\ Virginia Commonwealth University VCU Health, United States
}

Received: November 26, 2018

Accepted: January 4, 2019

Online Published: January 26, 2019

DOI: $10.5430 /$ jha.v8n1p44

URL: https://doi.org/10.5430/jha.v8n1p44

\begin{abstract}
Most acute care facilities are undergoing a major culture change and transforming into a high reliability organization focused on putting the patient experience first by delivering high quality, safe care. Reducing or eliminating hospital acquired conditions (HAC) fuels many quality improvement (QI) projects and successful reductions are attained when the support is rooted both in senior leadership and at the grassroots level. Yet each HAC requires a unique approach; specifically, pressure injury prevention programs have success with senior leaderships awareness and engagement in the complexities of the clinical and pathologic etiology of pressure injury development. This paper highlights for senior management the following evidence based key elements required for an organization to have a successful pressure injury prevention program: senior leadership engagement, a clinical champion, an interdisciplinary team, unit-based skin champions, nimble processes and access to evidence-based products.
\end{abstract}

Key Words: Pressure injuries, Pressure ulcers, Hospital acquired pressure ulcers, Hospital acquired pressure injuries, Pressure injury prevention, Pressure ulcer prevention, Unit-based champions

\section{INTRODUCTION}

Stemming from a pay for performance reimbursement shift, the banner flying above most health care systems today is one labeled with safety, quality, and customer service; putting the patient experience first. This is a paradigm shift from years past, when fee for service reimbursement forcedvolume as the key focus. Delivering safe, high quality care within a respectful, friendly atmosphere is a focus required by both payer sources and the public. ${ }^{[1]}$ In order to not only survive, but thrive in the competitive healthcare business, healthcare organizations have pivoted away from the perspective of quantity and volume towards one of quality. Safety, quality and customer service cannot be empty words; they must be worked into the culture and drive decision making on every level. Yet, even a loftier goal exists, namely becoming a high reliability organization.
In recent years, healthcare has taken on the transformational challenge to become a high reliability industry; which means consistent excellence in quality and safety for every patient, every time. High reliability in healthcare, according to The Joint Commission's Center for Transforming Healthcare, results in improved organizational effectiveness, efficiency, patient satisfaction, compliance, organizational culture, and documentation. ${ }^{[2]}$ It is a journey which impacts the breadth and depth of the organization and requires visionary, effective senior leaders to accept the challenge and map the journey. Leadership involvement in tackling and reducing hospital acquired conditions (HAC) is evidence of an organization's commitment to becoming a HRO. ${ }^{[3]}$

The purpose of this paper is to highlight the necessary support of senior leadership in reducing hospital acquired conditions;

*Correspondence: Sue Creehan; Email: sue.creehan@ vcuhealth.org; Address: Virginia Commonwealth University VCU Health, United States. 
specifically hospital-acquired pressure injuries (HAPI). The authors, all working on HAPI reduction for decades and seen as experts in the field of HAPI reduction, collectively shared input based on hands-on successful implementation of evidence-based comprehensive pressure injury prevention (PIP) programs in their organizations.

Throughout the article, current literature is cited and supports the basis of substantiating the senior leaders' influence and collaborative requirements to reach the goal of achieving an irreducible minimum.

\section{HAC REDUCTION - A LEADERS JOB}

Today it is no longer acceptable or financially reimbursable when patients are discharged from the hospital with an avoidable condition, or one that could "reasonably be avoided with the application of evidence based guidelines". ${ }^{[4]}$ HACs included: foreign object retained after surgery, air embolism, blood incompatibility, pressure ulcers (stage $3 \& 4$ ), injuries from falls and trauma, deep vein thrombosis and pulmonary embolism for total knee or hip replacement, poor glycemic control, iatrogenic pneumothorax, catheter-associated urinary tract infections, vascular catheter-associated infection, and surgical site infections (after coronary artery bypass, bariatric surgery, orthopedic surgery, and cardiac implants). ${ }^{[5]}$ Acute care facilities in the United States experience negative repercussions from such events through withholding of third party and government payments and the public reporting of metrics. HACs are all a safety issue, they are all a quality issue and they are all a customer service issue.

It is the responsibility of senior leadership to not only develop and articulate clear goals regarding HAC reduction, but to drive results. Driving results means leadership mobilizes people toward greater commitment to a vision, challenges people to set higher standards and goals, keeps people focused on achieving goals, gives direct and complete feedback that keeps teams and individuals on track, quickly takes corrective action as necessary to keep everyone moving forward, shows a bias toward action, and proactively works through performance barriers. ${ }^{[6]}$ In essence, driving results is a leader's way of creating a compelling call to action. To illustrate this point, consider this statement based on Greek history: "When Pericles spoke, people said, "how well he speaks." But when Demosthenes spoke, they said, "let us march." ${ }^{[7]}$

Likewise, transformational leadership is a style highly suitable for driving the results of HAC reduction. Transformational leadership is visible when the leader works with employees to identify the needed change, creates a vision to guide the change through inspiration, and executes the change in tandem with committed members of the group. Characteristics of a transformational leader include: stating a clear vision, connecting the board room to the bedside, supporting evidence-based practice, requiring data, encouraging staff participation, reviewing progress towards goals, removing identified barriers, establishing interdisciplinary teamwork and collaboration, and providing resources for staff. ${ }^{[8]}$

\section{Pressure injuries: Scope of the PROBLEM}

HAPIs are a significant healthcare problem, not only in the U.S., but across the globe. Patients who develop HAPIs experience pain, have a lower health-related quality of life, and are more likely to die during hospitalization. ${ }^{[9]}$ Annually, more than 2.5 million people in the U.S. develop a pressure injury ${ }^{[10]}$ at cost of $\$ 11$ billion. ${ }^{[11]}$ In 2007 when CMS was developing the non-payment policy for HACs, pressure ulcers were the highest volume and highest cost of all HACs. HAPIs are linked with higher rates of readmission, higher per patient cost, and increased length of stay, with a cost of $\$ 500-\$ 70,000$ per individual ulcer. ${ }^{[11]}$ Brem et al., (2010) suggest an estimated direct cost of $\$ 130,000$ for a single stage 3 or 4 HAPI case, not including the potential additional legal costs. ${ }^{[12]}$ In the United Kingdom, the National Health System developed a productivity calculator to help the government understand the productivity and cost elements associated with treating patients with pressure injuries.

\section{Pressure injury Prevention Lead- ERS}

The link between successful HAPI reduction and senior leadership support/engagement is validated in the literature through research, published guidelines and journal articles. Padula (2016) study HAPI rate reduction in 55 academic medical centers from 2007-2012. Common to the high performing HAPI reduction hospitals was 5 evidence based interventions, one of which was leadership engagement. ${ }^{[13]}$ In a systematic review of evidence regarding multicomponent strategies for pressure injury prevention and to examine the importance of contextual aspects of programs that aim to reduce HAPI, authors found 5 core components necessary, one of which is leadership involvement. ${ }^{[14]}$ Guidelines from the Agency for Healthcare Research and Quality (AHRQ), The Joint Commission (TJC), the Wound Healing Society (WHS), National Pressure Ulcer Advisory Panel (NPUAP), European Pressure Ulcer Advisory Panel (EPUAP) and Pan Pacific Pressure Injury Alliance (PPPIA), Wound Ostomy and Continence Nursing Society (WOCN), the Association for the 
Advancement of Wound Care (AAWC), and the Institute for Healthcare Improvement (IHI) provide evidence based approaches to systemwide HAPI prevention; each includes leadership initiatives as vital for success. Unfortunately, not all guidelines include the science of implementation, leaving healthcare groups on their own to digest, translate and implement the guidelines.

At the 2014 VCU Pressure Ulcer Summit, clinical experts and key stakeholders developed a framework for building a sustainable HAPI reduction program within an organization - leadership involvement, on a senior executive level, mid-level, and unit level was the first and most important element identified for success. ${ }^{[15]}$ Sage \& Tudor (2017) studied leadership rounding as a positive strategy, Dellefield and Magnabosco (2014) indicate clear statements by leadership that HAPI prevention is their priority and that reduction is possible but requires organizational culture change, and Frumenti \& Kurtz (2014) demonstrated HAPI reduction when mid-level leaders where educated and empowered to deal with operational failures and receive executive coaching from the Chief Nursing Officer (CNO). ${ }^{[16-18]}$ The volume of additional published documents noting the fundamental necessity of leadership involvement in HAPI reduction leaves no doubt that without it, success is unlikely.

\subsection{Executive leaders}

The senior leader most commonly overseeing HAPI reduction is the Chief Nursing Office (CNO) or the Performance or Quality Improvement manager; regardless, the key element is that this senior individual is identified and actively engaged in the challenge. Additionally, the senior finance leader must be supportive of the PIP program to advocate for capital expenditures, such as pressure redistribution beds or mattresses and other prevention expenses. PI treatment is costly, in capital and human resources, increased length of stay, higher morbidity and mortality, and human pain and suffering. Prevention also has a price tag, proven to be much less than treatment. ${ }^{[12,19]}$ The information technology leader is also linked into the PIP program through allocating resources to mine organizational and patient HAPI data. ${ }^{[20]}$ With all the competing demands on a leader's plate, one may ask: "Is it really necessary for senior leaders to be involved with pressure injuries?" The answer lies in the testimonials of successful organizations found in published literature.

Even for organizations in which leadership has been keenly involved in HAPI prevention initiatives, challenges prevail. In a qualitative study by Wald (2012), CNOs identified attribution and prevention as two ongoing challenges in their HAPI prevention programs. Attribution, or identification of the site of care where the patient acquired a PI, requires diligent admission assessment and documentation by both nursing and advanced practice provider or physician. Prevention struggles identified by CNOs were that some HAPIs may not be preventable despite exceptional nursing and medical care or no identified lapses in care and the frustration at bearing the burden of responsibility for unavoidable HAPIs as perceived failures by staff, the public and payors. ${ }^{[21]}$

Regardless of where an organization is on their HAPI reduction journey, leadership involvement is undeniably necessary for success and sustainability. Being positioned as a transformation leader to drive results is a high calling and is necessary in reducing HAPI, the most frequent and the costliest of all HACs.

In some organizations, the need for an improved PIP program begins with the clinicians at the bedside as an effort to improve quality care. Regardless of commencing as grassroots effort or a top-level administrative initiative, interdependence and team work is needed. When an organization takes on the commitment for HAPI prevention, senior leadership is undeniably necessary to support, build, and sustain a comprehensive PIP program. Basic ingredients of the program, discussed next, could be viewed as the 3 P's - people, process and products. The people or human resources necessary includes a clinical champion to lead the PIP program, an interdisciplinary team (IDT), and unit-based skin champions.

\subsection{Clinical champion leader}

In most hospitals, the identified PIP program champion is a certified nurse (Certified Wound Ostomy Continence Nurse - CWOCN, Certified Wound Care Nurse - CWCN, Certified Wound Ostomy Nurse - CWON, or Certified Wound Specialist - CWS), a clinical nurse specialist, or a performance improvement clinician. In a 2017 published study by Boyle, Bergquist-Beringer \& Cramer, out of 928 hospitals participating in the National Database of Nursing Quality Indicators (NDNQI), only one-third of them employed a certified wound nurse and each of their hospitals had less than half the number of HAPIs as the other hospitals. In addition, their findings suggest that the employment of a higher number of certified wound nurses is associated with decreased HAPI rates, improved pressure injury risk assessment, and improved prevention practices. ${ }^{[22]}$ The PIP champion's responsibilities may include but is not limited to: leading the IDT, data capture and analysis, improving processes, education, documentation, perhaps research, reporting to senior executives, and overseeing the unit-based skin champions. This individual's success will correlate to their ability to lead, effectively communicate, and their network of collaborative working relationships with key stakeholders such as Chief Financial Officer (CFO), Chief Procurement Officer or 
head of Materials Management, Chief Information Officer (CIO), physicians, advanced practice providers and mid-level managers. A comprehensive PIP program spans the breadth and depth of the organization, so connecting with all departments is imperative as the program grows. This includes the operating room, emergency department, interventional radiology, transportation, nutrition services, respiratory therapy, ancillary services, procedural areas etc.

\subsection{Interdisciplinary team leaders}

The responsibility for reducing HAPIs is frequently delegated to clinical teams with interdisciplinary (ID) representation. Senior leaders should look for role models who embrace the capacity to drive results and place these team members strategically on HAPI reduction ID teams. HAC reduction teams are more likely to be successful in accomplishing the goal when given not only the responsibility, but also the tools, resources, and appropriate level of authority to remove barriers and make decisions. Maintaining consistent communication with a senior leader liaison for bi-directional flow of information is also crucial for success.

ID practice or collaboration is described as a joint decisionmaking and communication process among health care providers that is patient centered, focusing on the unique needs of the patient and the specialized abilities of those providing care. Characteristics of ID collaboration include mutual respect, trust, good communication, cooperation, coordination, shared responsibility, and knowledge. ${ }^{[8]} \mathrm{Mem}-$ bers of the HAPI reduction ID team will vary from one organization to another, but a sample list is provided below (see Table 1).

Table 1. Suggested Interdisciplinary members serving on a HAPI Prevention Team

\begin{tabular}{ll}
\hline Members & Ad Hoc Members \\
Clinician & Ethics Representative \\
CNA/ nurse aide & Information Technology Representative \\
Dietician & Patient Mobility Team Respresentative \\
Direct Care Nurses & Materials Management Representative \\
Executive Leader & Nurse Educator \\
Physical Therapist & Patient/Family Advocate \\
Physician & Risk Management Representative \\
QI/PI Representative & \\
Respiratory Therapist & \\
Social Worker & \\
Transitional Care & \\
Representative/ & \\
Discharge Planner & \\
\hline
\end{tabular}

Published by Sciedu Press

\subsection{Unit-based skin champion leaders}

Pressure injury prevention must be translated from committee work to the touch point of care, at the bedside. Accountability for pressure injury prevention cannot lay solely on the shoulders of the clinical experts or members of the interdisciplinary team, it must be transferred to staff performing the interventions. Clinical staff respond positively and will modify their patient care delivery when influenced by peers. ${ }^{[8]}$ This peer to peer influence is most effectively accomplished through unit-based champions model. Staff nurses with a passion for quality and desire to learn more about wound care are ideal candidates for becoming a unit champion.

Unit based PIP champions are trained in the pathophysiology of PI, interventions for preventions, thorough visual skin assessments, accurate risk assessments and documentation. An example of their responsibilities may include; performing timely data collection, analyzing and bidirectional unit PI data reporting, attending meetings, role modeling PIP with each patient they care for, making PIP visible on their unit and educating their peers. A primary outcome of their work is to hold team members accountable as they work to drive down unit associated HAPI. The degree of support the unit manager provides the PIP champion is directly proportional to the champion's ability to be successful reducing the unit's PI. ${ }^{[23]}$ In addition to the people, processes and products are also relevant in reducing HAPIs.

\section{Processes}

There are many hospital processes in which PIP should be interwoven; clinical practice guidelines and pathways, decision algorithms, the electronic medical record (EMR) and education are a few. PIP begins on admission and follows the patient throughout the length of stay, so policies, procedures, guidelines, clinical practice standards, etc., must be evidencebased and comprehensive enough to impact care throughout the organization. PIP is not only a nursing responsibility; other members of the care team must understand and perform PIP interventions applicable to their role. This includes staff in ancillary areas such as emergency department, operating suite, dialysis, interventional radiology, transportation and other procedural areas. Since the science of PI teaches us a PI can occur in a short period of time with intense pressure, regardless of a patient's location during a hospital stay, staff must be engaged in prevention.

This comprehensive organizational commitment to PIP requires senior leadership support to hardwire processes in place such as empowering the Information Technology (IT) team to build effective EMR for PIP documentation. Leveraging the power of the EMR can improve adherence to best practices, optimize care planning, and customize event re- 
port feedback. ${ }^{[24]}$ Potential for data mining and warehousing can help organizations customize care practices for patients enabling efficient time management for health care providers. As hospitals focus on organizing data for review of HAPIs and other types of HACs, Padula et al., (2016) describe a "normalization process" that offers directions for collaboration between providers and informatics teams using a common language and structure. ${ }^{[13]}$

Senior leaders should support time and financial resources for adequate staff training on PIP. This may include mandatory initial and ongoing PIP staff education. Frequently voiced barriers to traditional education methods have been mitigated by technology. Technological advancements have made staff education amenable to multiple formats including remote learning, virtual classrooms, simulation labs, interactive web courses, and social media etc. Social media is an ideal format to create public awareness and interest in PIP.

\section{Products}

Effective PIP requires consistent application of evidencebased practice interventions utilizing a variety of products such as pressure redistribution surfaces, heel off-loading devices, moisturizing and barrier creams and prophylactic dressings. Similar to scientific evidence determining clinical practice guideline content, so should scientific evidence determine an organization's choice of products. PIP clinical leaders must stay up to date on published data and astutely assess the literature regarding product claims. For example, it has been proven both clinically and biomechanically that a specific 5 layer foam dressing can be used prophylactically to dramatically reduce sacral and heel pressure injuries. ${ }^{[25-27]}$

Expert healthcare economist, Dr. WilliamPadula published a six year observational cohort study looking at over 1 million patients and determined organizations that utilize a specific 5-layer foam dressing experienced an annual cost savings of $\$ 200,000-600,000 .{ }^{[19]}$ It is obvious that we all need to be good stewards over our organization's finances, but cutting corners and using generic products results in generic outcomes, not sustainable improved quality. Senior leaders should set a high standard for the acumen of value analysis team. True value analysis implies the financial and clinical voice hold equal weight in product decision making. [28]

Additionally, there is an investment required to ensure staff are trained and demonstrate immediate and ongoing competence in the use of PIP products. Staff also must have products immediately available at the point of care to ensure successful utilization.

\section{Conclusion}

The tide in healthcare is shifting. Quality, patient safety and customer service underpin the organizational culture change occurring in high reliability hospitals. Reducing avoidable harm such as hospital acquired pressure injuries requires engagement from the entire organization and focused support from senior management. Identifying key individuals for the role of clinical champion, interdisciplinary team members, and supporting unit-based skin champions are all a part of the formula for successful HAPI reduction. Application of evidence-based clinical practice guidelines laced with evidence-based products provide the best tools in decreasing avoidable harm and thwarting defense in litigation. Building a sustainable comprehensive organizational PIP program is the result of an intentional quest for quality.

\section{CONFlicts OF InTEREST Disclosure}

The authors declare they have no conflicts of interest.

\section{REFERENCES}

[1] American Association of Retired Persons. AARP Policy Book $2017-$ 2018; AARP health principles [Internet] [Accessed 2019 Jan 9]. Available from: https://policybook.aarp.org/policy-boo $\mathrm{k} /$ health/aarp-health-principles

[2] Joint Commission Center for Transforming Healthcare: Join the journey to high reliability [Internet] [cited 2018 Feb 7]. Available from: https://www. centerfortransformingheal thcare.or g/hro_portal_main.aspx

[3] Joint Commission Center for Transforming Healthcare: High reliability in healthcare is possible [Internet]. Available from: https ://www . centerfortransforminghealthcare.org/hi gh-reliability-in-health-care

[4] Centers for Medicare and Medicaid Services. Hospitalacquired conditions (present on admission indicator) (73 FR 48471-48491) [Internet]. [cited 2018 Feb 7]. Available from:
https://www.cms.gov/Medicare/Medicare-Fee-for-Ser vice-Payment/HospitalAcqCond/index.html

[5] Centers for Medicare and Medicaid Services. Hospitalacquired conditions ICD-10 HAC List. Available from: https ://www.cms.gov/Medicare/Medicare-Fee-for-S ervice-Payment/HospitalAcqCond/icd10_hacs.html

[6] Dye CF, Garman AN. Exceptional Leadership: 16 Critical Competencies for Healthcare Executives. Chicago, IL: Health Administration Press; 2006.

[7] Worthington I. Demosthenes of Athens and the Fall of Classical Greece. Oxford University Press: Williamsburg, VA; 2012.

[8] Roussel L. Management and leadership for nurse administrators. (5th ed). Sudbury, MA: Jones and Bartlett; 2009.

[9] Lyder C, Wang Y, Metersky M, et al. Hospital-acquired pressure ulcers: results from the national Medicare Patient Safety Monitor- 
ing System study. Journal of the American Geriatrics Society. 2012; 60(9): 1603-1608. PMid:22985136. https ://doi .org/10.1111/ j.1532-5415.2012.04106.x

[10] Agency for Healthcare Research and Quality. Preventing pressure ulcers in hospitals. A tool kit for improving quality of care [Internet]. [Cited 2018 Feb 9]. Available from: http://www.ahrq.gov/professionals/systems/hospital/ pressureulcertoolkit/index.html

[11] National Pressure Ulcer Advisory Panel, European Pressure Ulcer Advisory Panel and Pan Pacific Pressure Injury Alliance. Prevention and Treatment of Pressure Ulcers: Clinical Practice Guideline. Emily Haesler (Ed.). Cambridge Media: Perth, Australia; 2014.

[12] Brem H, Maggi J, Nierman D, et al. High Cost of Stage IV Pressure Ulcers. The American Journal of Surgery [Internet]. 2010: 473-477. https://doi.org/10.1016/j.amjsurg.2009.12.021

[13] Padula W, Blackshaw L, Brindle C, et al. An Approach to Acquiring, Normalizing, and Managing EHR Data From a Clinical Data Repository for Studying Pressure Ulcer Outcomes. JWOCN. 2016; 43(1): 39-45. https://doi .org/10.1097/WON. 0000000000000185

[14] Sullivan N, Shcoelles KM. Preventing in-facility pressure ulcers as a patient safety strategy: a systematic review. Ann Intern Med. 2013; 158(Pt 2): 410-6. PMid:23460098. https : //doi .org/10.7326/ 0003-4819-158-5-201303051-00008

[15] Creehan, et al. The VCU pressure ulcer summit - developing centers of pressure ulcer prevention excellence: a framework for sustainability. JWOCN. 2016 Mar; 42(3): 1-8.

[16] Sage S, Tudor H. Using positive clinical leadership to support a cutlrure of pressure injury prevention. Aust Nurs Midwifery J. 2017 Mar; 24(8): 39.

[17] Dellefield ME, Magnabosco JL. Pressure ulcer prevention in nursing homes: nurse descriptions of individual and organization level factors. Geriatric Nursing. 2014; 35: 97-104. PMid:24252559. https ://doi.org/10.1016/j.gerinurse.2013.10.010

[18] Frumenti JM, Kurtz A. Addressing hospital-acquired pressure ulcers. JONA. 2014 Jan; 44(1): 30-36. https : //doi .org/10 . 1097?NNA . 0000000000000018
[19] William P. The real-world effectiveness and value of sacral dressings to prevent hospital-acquired pressure injuries in academic medical centers: An observational cohort study. JWOCN. 2017 Sept; 44(5): 413-419. https://doi.org/10.1097/WON.0000000000000358

[20] Padula WV, Duffy MP, Yilmaz T, et al. Integrating systems engineering practice with health-care delivery. Health Syst. 2014; 3(3): 159-164. https://doi.org/10.1057/hs.2014.3

[21] Wald H, Richard A, Dickson VV, et al. Chief nursing officers' perspectives on Medicare's hospital-acquired conditions non-payment policy: implications for policy design and implementation. Implementation Science. 2012; 7: 78-89. PMid:22928995. https : //doi.org/10.1186/1748-5908-7-78

[22] Boyle D, Bergquist-Beringer S, Cramer E. Relationship of wound, ostomy, and continence certified nurses and healthcare-acquired conditions. JWOCN. 2017; 44(3): 283-292.

[23] Creehan S. Building nursing unit staff champion programs to improve clinical outcomes. Nurse Leader. 2015 Aug; 31-35. https : //doi.org/10.1016/j.mnl.2015.06.001

[24] Wang J, Gong Y. Potential of decision support in preventing pressure ulcers in hospitals. IOS Press; 2017. https : //doi .org/103233/9 78_I_61499-794-15

[25] Santamaria N, Liu W, Gerdtz MF, et al. The cost-benefit of using soft silicone multi-layered foam dressings to prevent sacral and heel pressure ulcers in trauma and critically ill patients: A within trial analysis of the Border Trial. Int Wound J. 2015; 12(3): 344-350. PMid:24750780. https://doi.org/10.1111/iwj.12160

[26] Santamaria N, O’Neil S, McCann J, et al. Clinical innovation: results from a five-year pressure ulcer prevention project in an Australian university hospital. Wounds International. 2015; 6(3): 6-10.

[27] Gefen A, Kottner J, Santamaria N. Clinical and biomechanical perspectives on pressure injury prevention research: the case of prophylactic dressings. J of Clin Biomechanics [Internet]. 2016 Aug [cited 2018 Mar 2]. https://doi.org/10.1016/j.clinbiomech. 20 16.08 .005

[28] Becker C. Calculating what it's worth. Value analysis pros making mark in healthcare. Modern Healthcare. July 2005; 35(27). 